J. Gastroenterol. Hepatol. Erkr. 2019· 17:59-67 https://doi.org/10.1007/s41971-019-0053-2

Online publiziert: 12. Juli 2019

(c) Der/die Autor(en) 2019

Margot Fodor ${ }^{1} \cdot$ Thomas Resch $^{1} \cdot$ Heinz Zoller ${ }^{2} \cdot$ Dietmar Öfner $^{1} \cdot$ Herbert Tilg $^{2}$. Dominik Wolf ${ }^{3}$ - Stefan Schneeberger ${ }^{1}$

' Universitätsklinik für Visceral-, Transplantations- und Thoraxchirurgie, Department für Operative Medizin, Medizinische Universität Innsbruck, Innsbruck, Österreich

${ }^{2}$ Universitätsklinik für Innere Medizin I, Gastroenterologie, Hepatologie und Endokrinologie, Department für Innere Medizin, Medizinische Universität Innsbruck, Innsbruck, Österreich

${ }^{3}$ Universitätsklinik für Innere Medizin V, Hämatologie und Internistische Onkologie, Department für Innere Medizin, Medizinische Universität Innsbruck, Innsbruck, Österreich

\title{
Lebertransplantation beim hepatisch metastasierten kolorektalen Karzinom: aktuelle Datenlage und mögliche Zukunftsszenarien
}

davon betreffen wiederum $50 \%$ die Leber [7]. Auch für eine mehrfache chirurgisch/radiologisch-interventionelle Sanierung ist ein Überlebensvorteil beschrieben [8]. Insbesondere bei bilobärer ausgedehnter Lebermetastasierung ist ein chirurgisches Vorgehen mittels etablierter Technologien jedoch häufig nicht möglich und die Option einer operativen Sanierung bleibt nur einer Minderheit dieser Patienten vorbehalten [9].

Das Vorliegen von nichtresektablen kolorektalen Metastasen (NRCRM) impliziert eine signifikant schlechtere Prognose. Als einzige therapeutische Option verbleibt die palliative CTx, die eine 5-JÜR von lediglich unter $10 \%$ ermöglicht [10]. Um eine Tumorprogression $\mathrm{zu}$ verhindern und/oder dennoch eine Resektabilität zu erreichen, werden häufig neoadjuvante CTx und lokaltherapeutische Verfahren angewendet. Grundsätzlich sollte die Vorgangsweise für alle $\mathrm{Pa}$ tienten mit Metastasen eines CRC in interdisziplinären Tumorboards bestimmt werden.

\section{Therapieoptionen}

Mehrere chirurgische sowie radiologisch-interventionelle Verfahren stehen zur Behandlung von CRC-Metastasen zur Verfügung. Kontrollierte Studien zum Vergleich zwischen den einzelnen Verfahren liegen gegenwärtig nicht vor. Die Entscheidung zum chirurgischen oder interventionellen Verfahren zur Sanierung von CRC-Metastasen erfolgt anhand etablierter Leitlinien. Im Detail obliegt die Therapieentscheidung bei ausgedehnten oder schwierig gelegenen Lebermetastasen jedoch einer Einschätzung in einem interdisziplinären Tumorboard.

\section{Lokoregionale Verfahren}

Die "perkutane Tumorablation“ ist ein minimal-invasiver Eingriff mit dem Ziel, Tumorgewebe durch lokale Gewebeschädigung zu zerstören. Dabei stehen verschiedene technische Verfahren zur Verfügung, denen gemeinsam ist, dass $\mathrm{Tu}-$ morzellen entweder durch Hitze oder Kälte destruiert werden. Die Radiofrequenzablation (RFA) verwendet hochfrequenten elektrischen Strom zur Erhitzung des Tumorgewebes, die Mikrowellenablation (MWA) hingegen hochfrequente Radiowellen. Während beide Verfahren auf Hitzeanwendung beruhen, wird bei der Kryoablation das Einfrieren von Gewebe angewandt. Die Rezidivrate Rezidivrate beträgt allerdings $40-75 \%$, 
beträgt bei RFA 5-60\%, das 5-JÜR variiert zwischen 17-51\%. Somit wird dieses Verfahren für eine limitierte Anzahl von Metastasen $(<5)$ mit einer Ausdehnung $<3 \mathrm{~cm}$ empfohlen [11]. Für die stereotaktische Multi-needle-RFA werden Lokalrezidivraten von $16 \%$ beschrieben [12].

Intraarterielle Therapien (IAT) kommen im Rahmen eines palliativen Settings bei Tumorprogression unter CTx zur Anwendung. Die Infusion von embolisierenden Agenzien, die selektiv in metastasenversorgende Leberarterien injiziert werden, ergänzt die systemisch verabreichte CTx und kann im Fall starker Nebenwirkungen eine Alternative darstellen. Die meist verwendeten Techniken sind die konventionelle transarterielle Chemoembolisation (cTACE), die „Drug-eluting bead (DEB-)TACE“ und die selektive interne Strahlentherapie (SIRT). Eine Übersichtsanalyse zeigte Ansprechraten nach den RECISTKriterien von 23,36 und $23 \%$, während die mediane Überlebensraten ab Ersttherapie enttäuschende 16, 16 und 12 Monate ergaben [11].

\section{Chirurgische Verfahren}

Im Fall vorhandener Resektabilität und ausreichender verbleibender Restleber gilt die chirurgische Resektion als Methode der Wahl. Die Kriterien der Resektion entsprechen den allgemeinen Prinzipien der Leberchirurgie, weshalb hier auf eine detaillierte Beschreibung verzichtet wird.

Schwieriger gestaltet sich die Situation im Fall eines zu erwartenden unzureichenden Leberrestvolumens. Moderne Therapiestrategien ermöglichen in ausgewählten Fällen auch hier die Anwendung von Chirurgie in kurativer Intention. So kann bei Tumorfreiheit der Segmente II und III der Leber ein zweizeitiges Vorgehen gewählt werden, wobei zunächst eine Portalvenenembolisation (PVE) durchgeführt wird, um in weiterer Folge eine Volumenzunahme der zukünftigen Restleber herbeizuführen. Hierbei kann eine Volumenzunahme von $27-39 \%$ in den ersten 4-8 Wochen erreicht werden [13]. In einer zweiten Sitzung wird die Resektion der Segmente (I und) IV-VIII durchgeführt. Trotz guter Erfolge dieses neuen Therapieregimes verbleibt das Risiko einer Tumorprogression oder einer insuffizienten Hypertrophie während des Intervalls, wodurch eine endgültige Resektion in $25-38 \%$ der Fälle nicht durchgeführt werden kann [13]. Im Fall einer unzureichenden Volumenzunahme der zukünftigen Restleber kann ein chirurgischer Verschluss der Portalvenen für die Segmente IV-VIII im Rahmen des Associating-liver-partitionand-portal-vein-ligation-for-staged-hepatectomy(ALPPS)-Verfahrens durchgeführt werden. Durch diese Methode kann ein stärkeres Wachstum an Leberrestvolumen in einem kürzeren Zeitintervall induziert werden. Limitierend bleiben hier jedoch hohe Komplikationsraten mit entsprechender Morbidität und Mortalität, die mit 68 und $12 \%$ angegeben werden [13]. Vergleichsanalysen für PVE und ALPPS konnten letztlich ähnliche 90-Tage-Mortalitätsraten (8\%) zeigen [14]. Wegen der besseren Wachstumsrate im Rahmen der ALPPSProzedur wird diese heute vorrangig bei unzureichender Volumenzunahme trotz Embolisation angewendet. Große randomisierte Studien, die die Effizienz der PVE jener der ALPPS-Prozedur gegenüberstellen, sind zum gegenwärtigen Zeitpunkt jedoch noch ausständig [13].

Trotz der empfohlenen Standardtherapien, der zusätzlichen erweiterten chirurgischen Optionen und der möglichen lokoregionalen Verfahren gibt es für Patienten mit multiplen bilobären Lebermetastasen derzeit keine gute therapeutische Alternative. Um diesen Patienten helfen zu können, wurde in den letzten Jahren neuerlich die Lebertransplantation (LTX) als Option für Patienten mit NRCRM beleuchtet. Mittels Lebertransplantation können zufriedenstellende Ergebnisse für Malignome, wie das hepatozelluläre Karzinom (HCC), ausgewählte Fälle des Cholangiokarzinoms sowie für hepatale Metastasen einer neuroendokrinen Neoplasie (NEN), erzielt werden [8]. Die Spenderknappheit sowie nicht überzeugende Ergebnisse aus einer ersten Studie stellen jedoch eine Hürde für die LTX bei NRCRM dar [8].

\section{Lebertransplantation}

\section{Frühe Analysen}

Die ersten Daten hinsichtlich LTX bei CRM stammen aus einer Wiener Serie. In einer Kohorte aus 25 Patienten, die zwischen 1982 und 1994 transplantiert wurden, betrug die 1-, 3- und 5-JÜR jeweils 76,32 und $12 \%$ und blieb somit weit hinter den Erwartungen zurück. Diese schlechten Ergebnisse wurden in der Folge als Begründung herangezogen, eine LTX nicht für die Indikation NRCRM heranzuziehen. Allerdings relativiert sich diese Aussage bei genauerer Betrachtung der Originalarbeiten. Die 30-Tage-Mortalität lag mit $30 \%$ sehr hoch, was nicht der Grundkrankheit CRM geschuldet ist, sondern der zu diesem Zeitpunkt hohen Mortalität nach $\operatorname{LTX}[15,16]$. Zu den Einschlusskriterien gehörte ein negativer Lymphknotenstatus. Im Rahmen einer Folgeuntersuchung konnte jedoch gezeigt werden, dass von 21 Patienten mit vermeintlich tumorfreien Lymphknoten letztlich 15 Mikrometastasen aufwiesen. Der signifikante Überlebensnachteil solcher Patienten mit Mikrometastasen gegenüber jenen Patienten ohne Mikrometastasen bietet eine Erklärung für die insgesamt unzureichenden Ergebnisse (28 vs. 118 Monate; [17]). Allerdings wiesen auch einige weitere kleinere Serien aus den frühen 1990er-Jahren keine überzeugenden Ergebnisse für die LTX bei NRCRM auf. So wurde in einer 1991 veröffentlichten Arbeit eine heterogene Patientengruppe mit LTx bei 8 NRCRM-Patienten beschrieben. Die Rezidivrate betrug $70 \%$, die 2- und 5-JÜR jeweils 38 und $21 \%$ [18]. Laut dem europäischen LTXRegister (ELTR) wurden zwischen 1977 und 1995 insgesamt 58 Fälle von LTX bei NRCRM durchgeführt. Die dabei erreichten 1-, 3- und 5-JÜR betrugen 73, 36 und $18 \%$ [19]. Zusammenfassend kann, auch nach Einbeziehen einiger relativierender Einflussfaktoren, festgestellt werden, dass die LTX bei NRCRM vor dem Jahr 2000 insgesamt nur wenig vielversprechende Ergebnisse erzielen konnte $[8,20]$.

Weitere Versuche, die LTX bei CRCRM zu etablieren, wurden unterlassen, bis eine Gruppe aus Oslo dieses Thema 
Hier steht eine Anzeige.

\section{曾 Springer}




\section{Lebertransplantation beim hepatisch metastasierten kolorektalen Karzinom: aktuelle Datenlage und mögliche Zukunftsszenarien}

\section{Zusammenfassung}

Das kolorektale Karzinom (KRK) ist die weltweit dritthäufigste Krebserkrankung. Zirka $20 \%$ der Patienten präsentieren sich bei der Diagnose mit Lebermetastasen, zirka 30\% entwickeln im Verlauf Lebermetastasen. Die chirurgische Sanierung, häufig in Kombination mit einer systemisch wirksamen Chemotherapie, stellt in aller Regel die einzige kurative Therapie dar. Allerdings ist aufgrund des oft unzureichenden postoperativ verbleibenden Leberrestvolumens nur ein Teil der Patienten mit hepatalen kolorektalen Metastasen (CRM) für eine Resektion geeignet. Insbesondere eine bilaterale, diffuse Lebermetastasierung macht eine chirurgische Sanierung bisher oft unmöglich. In den letzten Jahren wurde eine Vielfalt an erweiterten chirurgischen Maßnahmen und lokoregionalen Therapien für primär nichtresektable oder marginal resektable kolorektale Metastasen (NRCRM) entwickelt. Während mit alleiniger chirurgischer Resektion 5-Jahres-Überlebensraten (JÜR) um $20 \%$ erreicht wurden, konnten diese durch neue neoadjuvante und adjuvante Therapien sowie Fortschritte in der chirurgischen Technik deutlich verbessert werden. Eine Lebertransplantation (LTX) stellt nun eine attraktive Alternative für fitte Patienten mit (nichtresektablen) NRCRM ohne extrahepatische Beteiligung dar. Rezente Studien attestieren diesem Ansatz 5-JÜR von über $50 \%$. Diesen medizinischen Erfolgen zum Trotz ist aufgrund der Organknappheit an eine LTX als Standardvorgehen zur Behandlung von NRCRM nicht zu denken. Durch eine neue Technik, bei der nur ein kleiner Teil einer Spenderleber für NRCRMPatienten transplantiert wird, ergibt sich nun ein neuartiger und innovativer Lösungsansatz. Dieser Übersichtsartikel veranschaulicht die Hintergründe dieses derzeit in Studien getesteten und potenziell kurativen Therapieverfahrens bei NCRCM-Patienten und beleuchtet kritisch diese neuartigen und hoffnungsvollen Entwicklungen, die die Onkologie und Transplantationsmedizin möglicherweise nun auch über das hepatozelluläre Karzinom (HCC) hinaus verbinden wird.

\section{Schlüsselwörter}

Transplantation · Onkologie · Nichtresektable kolorektale Metastasen - Maschinenperfusion . Ethik

\section{Liver Transplantation for Colorectal Liver Metastasis: Current Data and Possible Future Scenarios}

\section{Abstract}

Colorectal cancer (CRC) is the third most common cancer worldwide. The majority of CRC patients will develop distant metastases during the course of their disease, most of them occurring in the liver. To date, liver resection represents the only curative option. However, due to insufficient liver remnant volume, only a minority of patients with hepatic CRC metastasis are candidates for liver resection. In recent years, various surgical and minimally invasive procedures have been developed for the treatment of non-resectable colorectal liver metastases
(NRCRM). Whereas initial reports yielded 5 -year overall survival (OS) rates of less than $20 \%$, results have now significantly improved owing to the ameliorated chemotherapy and refined surgical techniques. Furthermore, liver transplantation (LTX) has surfaced as a promising option for patients with NRCLM. Recent studies report 5-year OS rates exceeding 50\%. Despite this success, wider application of LTX for the treatment of NCRLM is currently hampered by the persisting organ shortage. This ethical dilemma may now be overcome through innovative protocols which allow the use of only a small part of the donor liver for NRCLM patients while the remaining part of the graft is transplanted to a second recipient. This review illustrates the underlying factors which have so far hindered a curative approach for NRCRM patients and highlights recent promising achievements in oncology and transplant medicine.

\section{Keywords}

Transplantation - Oncology · Non-resectable colorectal metastases - Machine perfusion . Ethics rezent wieder aufgriff, um diesen experimentellen und innovativen Therapieansatz unter kontrollierten Bedingungen neu zu evaluieren. In einer ersten prospektiven Studie, dem SECA Trial, wurde das Überleben nach LTX bei CRM unter modernen Bedingungen überprüft. Hilfreich für die Machbarkeit der Studie war für die Osloer Autoren in diesem Zusammenhang, dass in Norwegen aufgrund der hohen Spenderzahlen eine gute Versorgungssituation mit Spenderorganen mit Wartezeiten von weniger als einem Monat bis zur LTX bestehen. Einschlusskriterien für diese Studie waren eine R0-Resektion des Primärtumors, mindestens 6 Wochen CTx, NRCRM, keine weitere Fernmetastasierung und ein ECOG-Status 0-1. Patienten wurden einer diagnostischen Laparotomie mit Biopsie der Lymphknoten im Leberhilus unterzogen. Sofern sich kein Hinweis für Lymphknotenmetastasen ergab, wurde eine LTX durchgeführt. Es konnten insgesamt 21 Patienten transplantiert werden. Es wurde keine präoperative oder adjuvante CTx verabreicht, die immunsuppressive Therapie wurde mit Sirolimus durchgeführt. Die 1-, 3- und 5-JÜR waren jeweils 95, 68 und
$60 \%$ (• Abb. 1). Die Rezidivrate betrug $90 \%$. Sieben Patienten entwickelten ein singuläres pulmonales, weitere 7 ein hepatales Rezidiv [21]. Dieselbe Arbeitsgruppe untersuchte in weiterer Folge die Rezidivmuster in dieser Kohorte. Das durchschnittliche rezidivfreie Intervall betrug 6 Monate. Die Erstmanifestation war in den meisten Fällen die Lunge (13/21, 7 singulär; • Abb. 2). Von diesen konnten 3 Patienten reseziert werden, die restlichen erhielten lokoregionale Therapieverfahren und/oder CTx. Nach 27 Monaten waren alle Patienten mit singulären Lungenmetastasen noch am 


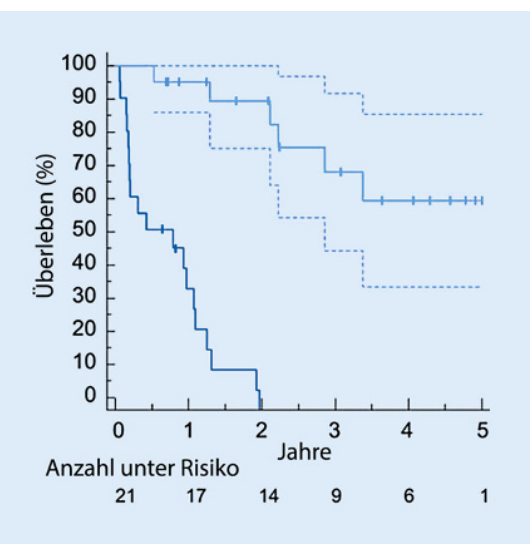

Abb. $1 \Delta$ SECA Trial: 1-, 3- und 5-Jahresüberlebensraten (JÜR) waren jeweils 95,68 und $60 \%$. (Mit Genehmigung aus: Hagness et al. [21])

Leben. Das hepatale Rezidiv hingegen ereignete sich immer in Kombination mit einer zusätzlichen Fernmetastasierung, wobei die Leber nie als Organ der Rezidiverstmanifestation festgestellt wurde. Die 5-JÜR bei pulmonaler Metastasierung betrug $72 \%$, sodass die Autoren konkludierten, dass diese weniger Einfluss auf das Überleben nach LTX hat. Im Vergleich dazu konnten für multiple Lungen- und Leberresektionen bei CRM 5-JÜR von $45 \%$ erzielt werden [22]. Im Jahr 2005 verglich die Osloer Arbeitsgruppe die SECA-TrialKohorte $(n=21)$ mit der NORDIC-VIITrial-Kohorte $(n=47)$. In der NORDICVII-Trial-Kohorte wurden verschiedene CTx-Kombinationen bei Patienten mit hepatalen nicht-BRAF-mutierten NRCRM verabreicht: Fluorouracil/Folsäure plus Oxaliplatin (FOLFOX) als Bolus, FOLFOX plus Cetuximab und intermittierend verabreichtes FOLFOX plus Cetuximab. Im direkten Vergleich zwischen SECA und NORDIC VII Trial zeigten sich jeweils eine 5-JÜR von $56 \%$ vs. $19 \%$ (• Abb. 3) sowie eine mediane Tumorfreiheit von 10 Monaten (SECA Trial, $n=21$ ) und 8 Monaten (NORDIC VII Trial, $n=47$; • Abb. 4; [23]).

Rezent wurde eine weitere Serienanalyse aus 12 Patienten nach LTX bei NRCLM und einem Follow-up von 41 Monaten nach primärer Resektion veröffentlicht [22]. Bei 11 der Patienten wurden präoperativ Irinotecan oder Oxaliplatin verabreicht. Die 1-, 3- und 5-JÜR betrug jeweils 83, 62 und 50\%.

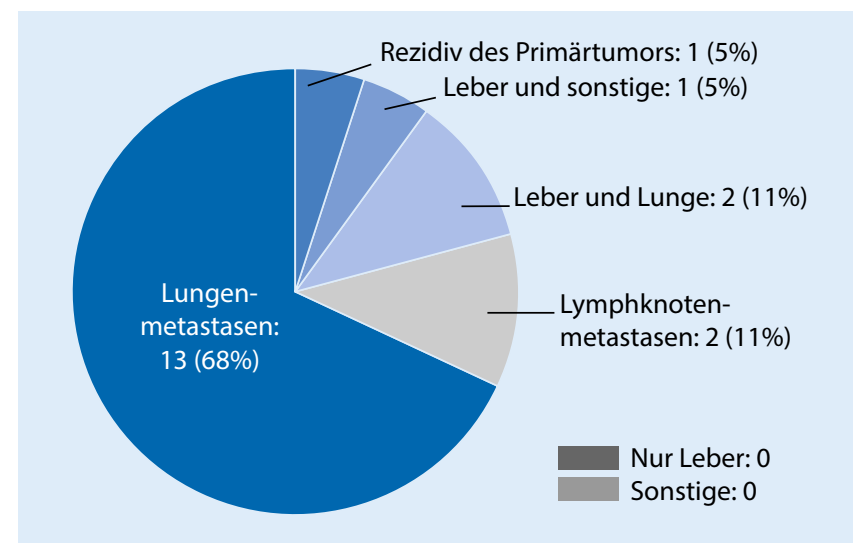

Abb. $2 \triangleleft$ SECA

Trial: Rezidivmuster nach LTX. (Aus [24])
Sechs Patienten erlitten ein Rezidiv, 5 davon in der Lunge. Bemerkenswerterweise konnte ein rezidivfreies Intervall von 48 Monaten erreicht werden [26].

Bei tendenziell schlechter 5-JÜR $(<20 \%)$ und hohem Rezidivrisiko wurde also initial die LTX als kurative Intention bei NRCRM unterlassen. Allerdings zeigten sich in neueren Studien sowohl durch genauer definierte Selektionskriterien als auch durch Verbesserung in den Bereichen Chirurgie, CTx und Immunsuppression nun deutlich bessere 5-JÜR (>50\%). Die immer noch hohe Rezidivrate ist relevant, jedoch kann durch Resektion, interventionelle Therapie und adjuvante Therapie ein längerfristiges Überleben auch in dieser Situation erreicht werden.

\section{Status quo und laufende Studien}

Bei unveränderter Diskrepanz zwischen Organbedarf und Organangebot mit weiterhin bestehender Mortalität auf der Warteliste wurden in den letzten Jahren auch ältere Spender sowie Spender mit vermehrt zusätzlichen Komorbiditäten utilisiert. Weiters werden zunehmend „non-heart-beating“ Spender, Splitlebern oder Organe von Lebendspendern für die Transplantation verwendet [8]. Im Zuge der Allokation werden Organe primär an Patienten mit der höchsten Dringlichkeit im Sinne einer Lebererkrankung mit Funktionsverlust vergeben. Bei Patienten mit NRCRM besteht selten ein Leberfunktionsverlust und die Zuteilung einer Leber an diese Patienten ist nach den derzeitigen Kriterien nur dann möglich, wenn diese Leber für keine Patienten mit höherem MELD-Score verwendet werden kann. Die Lebendspende stellt eine mögliche alternative Organressource dar und ist zuletzt als Alternative für NRCRM evaluiert worden [8]. Die Vorteile liegen hierbei auf der Hand, nämlich besteht neben einer deutlich kürzeren Wartezeit die Möglichkeit, eine Operation genau zu planen. Vor einer LTX können sowohl ein umfassendes Staging als auch eine präoperative CTx komplettiert werden. Diese Vorteile werden aktuell in einer laufenden kanadischen Studie untersucht, in der NRCRM-Patienten nach erfolgter 6-monatiger CTx einer Lebendspende unterzogen werden. Erste Ergebnisse sollen 2020 vorgestellt werden [8].

Weitere laufende Studien zur Evaluation von LTX bei NRCRM sind die SECA-II-Studie, die RAPID-Studie und die TRANSMET-Studie. Die SECA-II-Studie ist eine kontrollierte randomisierte Analyse zur Evaluierung der Überlebensrate bei Patienten mit NRCRM, die entweder einer LTX oder einer Resektion unterzogen wurden. Die Ergebnisse sollen 2027 veröffentlicht werden [23]. Dieselbe Arbeitsgruppe etablierte auch das Konzept der „resection and partial liver segment 2-3 transplantation with delayed total hepatectomy“ (RAPID), bei dem eine Resektion und Transplantation der Lebersegmente II und III in einem ersten Schritt durchgeführt wird. Die Ent- 


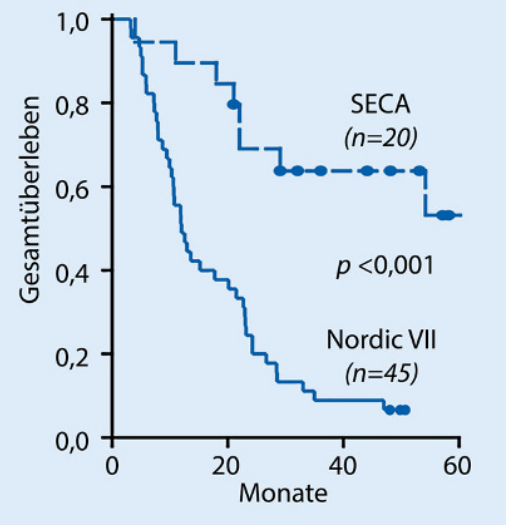

Abb. 3 ॥ Vergleich SECA/NORDIC VII Trial, 5-JÜR von $56 \%$ vs. $19 \%$ [23]. (Mit Genehmigung aus: Dueland et al. [25])

fernung der zunächst verbliebenen tumorbefallenen Segmente (I und) IV-VIII wird nach Erreichen eines adäquaten Volumens der transplantierten Segmente II und III durchgeführt. Diese Hypertrophie der transplantierten Segmente wird durch eine Verminderung der Blutzufuhr zur Restleber erreicht (in Analogie zur ALPPS-Prozedur). Der fundamentale Unterschied zur Transplantation einer ganzen Leber bei NRCRM besteht darin, dass die verbleibende Leber (Segmente I, IV-VIII) an einen weiteren Patienten transplantiert werden kann. Dieses Konzept ermöglicht auch die Durchführung einer Lebendspende der Segmente II und III, wobei das Risiko für den jeweiligen Spender mit einem Mortalitätsrisiko von $0,1 \%$ äußerst gering einzustufen ist. Die Studie soll die Sicherheit und den Überlebensvorteil bei sekundärer Hepatektomie 4 Wochen nach LTX der Segmente II-III beurteilen. Während die letzten Ergebnisse der Originalstudie 2028 erwartet werden können [8], wird das Konzept nun auch in anderen Zentren inklusive der Medizinischen Universität Innsbruck angeboten.

Die TRANSMET-Analyse rekrutiert in einer randomisierten Studie Patienten mit NRCRM für Standard-CTx oder LTX plus CTx. Hier werden 2027 erste Ergebnisse erwartet [8]. Auch wenn aus wissenschaftlicher Sicht die Randomisierung sicher dem Goldstandard entspricht, ist diese bei NRCRM schwierig [27]. Die zunehmend guten Ergebnisse suggerieren einen Überlebensvorteil für

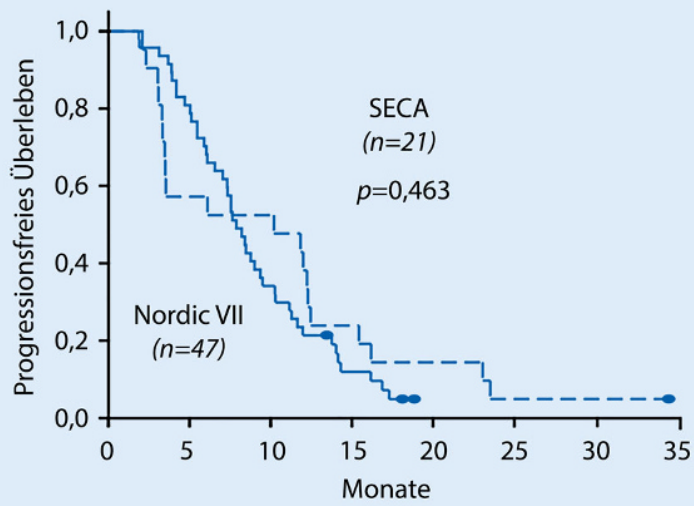

Abb. $4<$ Vergleich SECA/NORDIC VII Trial, mediane Tumorfreiheit von 10 Monaten (SECA Trial) vs. 8 Monaten (NORDIC VII Trial). (Mit Genehmigung aus: Dueland et al. [25]) diese Patienten durch eine LTX. Durch Abwarten der Ergebnisse der laufenden prospektiven Analysen wird diesen Patienten womöglich eine bessere Therapie vorenthalten [8].

\section{Ausblick}

Organe von hirntoten Spendern mit „erweiterten Kriterien“ können und müssen in der Transplantation verwendet werden, um dem Bedarf gerecht zu werden. Perioperativ werden diese Organe durch Unterbrechung der Nähr- und Sauerstoffzufuhr zusätzlich geschädigt. Rezente Studien suggerieren, dass physiologische Konservierungsbedingungen von Lebern durch Maschinenperfusion (MP) die Organe vor Schädigungen schützen und somit eine valide Alternative zur klassischen statischen Kaltlagerung (,static cold storage“ [SCS]) darstellen. Vorteil der MP ist einerseits die Möglichkeit, die Organqualität vor der LTX (ex situ) zu testen und diese Organe (in der Zukunft) zu rekonditionieren und deren Funktion zu verbessern. Eine Anzahl von mehr als 500 experimentellen und klinischen Forschungsarbeiten wurde in den letzten 10 Jahren zu diesem Thema publiziert. Grundsätzlich wird zwischen hypother$\operatorname{mer}\left(0-12^{\circ} \mathrm{C}\right)$, mittelgradiger $\left(13-24^{\circ} \mathrm{C}\right)$, subnormothermer $\left(25-34^{\circ} \mathrm{C}\right)$ und normothermer $\left(35-38^{\circ} \mathrm{C}\right) \mathrm{MP}$ differenziert. Der Temperaturunterschied beeinflusst den Organmetabolismus und damit auch Konservierung und Funktion. Die MP wird je nach Technik zu unterschiedlichen Zeitpunkten durchgeführt: a) gleich nach Organentnahme, b) vor dem Transport auf Eis (prästatische Kaltlagerung)/ kurz vor Transplantation (poststatische Kaltlagerung) oder c) während der Gesamtzeit zwischen Organentnahme und Transplantation des Organs [28].

Die normotherme Maschinenperfusion (NMP) basiert auf einem Blutperfusat und konserviert Organe unter nahezu physiologischen Bedingungen, zusätzlich können eine Organkonditionierung und Monitorisierung der Viabilität/ Funktion durchgeführt werden [29, 30].

Daten der ersten kontrollierten PhaseIII-Studie zur NMP bei Lebern ergab, dass das Risiko für eine frühe Dysfunktion des Organs nach NMP - trotz längerer Konservierungszeit (11,9h vs. 7,75h) um $74 \%$ reduziert werden konnte [31]. Diese und weitere Ergebnisse suggerieren, dass die Organkonservierung einen zentralen Baustein in der Bemühung, dem Mangel an Organspendern entgegenzuwirken, darstellt. Durch eine Erweiterung des Spenderpools kann eine Indikationserweiterung der LTX vor allem für onkologische Indikationen möglich werden.

\section{Fazit für die Praxis}

- Das kolorektale Karzinom (KRK) ist weltweit die dritthäufigste Krebserkrankung, die trotz verbesserter Therapieoptionen durch hohe Mortalität gekennzeichnet ist. 
Hier steht eine Anzeige.

\section{曾 Springer}




\begin{tabular}{|c|c|}
\hline \multicolumn{2}{|c|}{ Abkürzungen } \\
\hline ALPPS & $\begin{array}{l}\text { "Associating liver partition and } \\
\text { portal vein ligation for staged } \\
\text { hepatectomy" }\end{array}$ \\
\hline CRM & Kolorektale Metastasen \\
\hline$C T$ & Chemotherapie \\
\hline CTACE & $\begin{array}{l}\text { Konventionelle transarterielle } \\
\text { Chemoembolisation }\end{array}$ \\
\hline$D C D$ & „Donation after cardiac death" \\
\hline DEB-TACE & "Drug-eluting bead TACE" \\
\hline FLOX & $\begin{array}{l}\text { Fluorouracil/Folsäure plus } \\
\text { Oxaliplatin }\end{array}$ \\
\hline $\mathrm{HCC}$ & Hepatozelluläres Karzinom \\
\hline$I A T$ & Intraarterielle Therapien \\
\hline KRK & Kolorektales Karzinom \\
\hline$L T X$ & Lebertransplantation \\
\hline$M P$ & Maschinenperfusion \\
\hline$M W A$ & Mikrowellenablation \\
\hline NEN & Neuroendokrine Neoplasie \\
\hline NMP & $\begin{array}{l}\text { Normotherme Maschinenperfusi- } \\
\text { on }\end{array}$ \\
\hline$N R C R M$ & $\begin{array}{l}\text { Nichtresektable kolorektale } \\
\text { Metastasen }\end{array}$ \\
\hline$P V E$ & Portalvenenembolisation \\
\hline RAPID & $\begin{array}{l}\text { "Resection and partial liver } \\
\text { segment } 2 / 3 \text { transplantation with } \\
\text { delayed total hepatectomy" }\end{array}$ \\
\hline RFA & Radiofrequenzablation \\
\hline SCS & "Static cold storage" \\
\hline SIRT & "Selektive interne Radiotherapie" \\
\hline
\end{tabular}

- Ungefähr die Hälfte aller Patienten mit KRK entwickelt im Krankheitsverlauf Lebermetastasen.

- Der Einsatz multimodaler Therapiekonzepte inklusive der Konversionschemotherapie zur sekundären Resektablilität stellt auch bei ausgedehnter hepatischer Metastasierung keine Kontraindikation für potenziell kurative Behandlungsansätze dar.

- State-of-the-Art-Therapie bei resektablen Metastasen ist aktuell die Kombination aus Resektion und (neo-)adjuvanter CTx.

- Bei NRCRM gilt die palliative CTx mit 5-JÜR von $<10 \%$ als Standard; weitere Optionen sind erweiterte chirurgische Maßnahmen und lokale therapeutische Verfahren.

- Die LTX stellt eine vielversprechende Option für Patienten mit NRCRM, der aktuelle Organmangel stellt das größte Hindernis zur Indikationserweiterung dar.

\section{Korrespondenzadresse}

\section{ao. Univ.-Prof. Dr. Stefan Schneeberger,} Executive MBA HSG

Universitätsklinik für Visceral-, Transplantations- und Thoraxchirurgie, Department für Operative Medizin, Medizinische Universität Innsbruck

Anichstraße 35, 6020 Innsbruck, Österreich

Stefan.Schneeberger@i-med.ac.at

Funding. Open access funding provided by University of Innsbruck and Medical University of Innsbruck.

\section{Einhaltung ethischer Richtlinien}

Interessenkonflikt. M. Fodor, T. Resch, H. Zoller, D. Öfner, H. Tilg, D. Wolf und S. Schneeberger geben an, dass kein Interessenkonflikt besteht.

Für diesen Beitrag wurden von den Autoren keine Studien an Menschen oder Tieren durchgeführt. Für die aufgeführten Studien gelten die jeweils dort angegebenen ethischen Richtlinien.

Open Access Dieser Artikel wird unter der Creative Commons Namensnennung 4.0 International Lizenz (http://creativecommons.org/licenses/by/4.0/deed. de) veröffentlicht, welche die Nutzung, Vervielfältigung, Bearbeitung, Verbreitung und Wiedergabe in jeglichem Medium und Format erlaubt, sofern Sie den/die ursprünglichen Autor(en) und die Quelle ordnungsgemäßnennen, einen Linkzur Creative Commons Lizenz beifügen und angeben, ob Änderungen vorgenommen wurden.

\section{Literatur}

1. Ferlay J, Soerjomataram I, Dikshit R, Eser S, Mathers C, Rebelo Met al (2015) Cancer incidence and mortality worldwide: sources, methods and major patterns in GLOBOCAN 2012. Int J Cancer 136(5):E359-E586

2. Hagness M (2015) Liver transplantation in treatment of colorectal liver metastases. Hepat Oncol 2(2):181-190

3. Statistik Austria 2019. https://www.statistik. at/web_de/statistiken/index.html. Zugegriffen: 04.07.2019

4. van der Geest LG, Lam-Boer J, Koopman M, Verhoef C, Elferink MA, de Wilt JH (2015) Nationwide trends in incidence, treatment and survival of colorectal cancer patients with synchronous metastases. Clin Exp Metastasis 32(5):457-465

5. van Gestel YR, de Hingh IH, van Herk-Sukel MP, van Erning FN, Beerepoot LV, Wijsman JH et al (2014) Patterns of metachronous metastases after curative treatment of colorectal cancer. Cancer Epidemiol 38(4):448-454

6. Benson $A B$, Bekaii-Saab $T$, Chan $E$, Chen $Y J$, Choti MA, Cooper HS et al (2013) Metastatic colon cancer, version 3.2013: Featured updates to the NCCN guidelines. J Natl Compr Canc Netw 11(2):141-152 (quiz 152)

7. Bredt LC, Rachid AF (2014) Predictors of recurrence after a first hepatectomy for colorectal cancer liver metastases: a retrospective analysis. World J Surg Oncol 12:391

8. Gorgen A, Muaddi H, Zhang W, McGilvray I, Gallinger S, Sapisochin G (2018) The new era of transplant oncology: liver transplantation for nonresectable colorectal cancer liver metastases. Can J Gastroenterol Hepatol. https://doi.org/10 1155/2018/9531925

9. Pulitano C, Crawford M, Joseph D, Aldrighetti L, Sandroussi C (2014) Preoperative assessment of postoperative liver function: the importance of residual liver volume. J Surg Oncol 110(4):445-450

10. Sanoff HK, Sargent DJ, Campbell ME, Morton RF, Fuchs CS, Ramanathan RK et al (2008) Fiveyear data and prognostic factor analysis of oxaliplatin and irinotecan combinations for advanced colorectal cancer: N9741. J Clin Oncol 26(35):5721-5727

11. Chow FC, Chok KS (2019) Colorectal liver metastases: an update on multidisciplinary approach. World J Hepatol 11(2):150-172

12. Bale $R$, Widmann $G$, Schullian $P$, Haidu $M$ Pall G, Klaus A et al (2012) Percutaneous stereotactic radiofrequency ablation of colorectal liver metastases. Eur Radiol 22(4):930-937

13. Sandström P, Røsok BI, Sparrelid E, Larsen PN, Larsson AL, Lindell G et al (2018) ALPPS improves resectability compared with conventional twostage hepatectomy in patients with advanced colorectal liver metastasis: results from a Scandinavian multicenter randomized controlled trial (LIGRO trial). Ann Surg 267(5):833-840

14. Shindoh J, Vauthey JN, Zimmitti G, Curley SA, Huang SY, Mahvash A et al (2013) Analysis of the efficacy of portal vein embolization for patients with extensiveliver malignancyand verylow future liver remnant volume, including a comparison with the associating liver partition with portal vein ligation for staged hepatectomy approach. J Am Coll Surg 217(1):126-133 (discussion 133-134)

15. Mühlbacher $F$, Huk I, Steininger $R$, Gnant $M$, Götzinger P, Wamser P et al (1991) Is orthotopic liver transplantation a feasible treatment for secondary cancer of the liver? Transplant Proc 23(1 Pt 2):1567-1568

16. Mühlbacher F, Piza F (1987) Orthotopic liver transplantation for secondary malignancies of the liver. Transplant Proc 19(1 Pt3):2396-2398

17. Kappel S, Kandioler D, Steininger R, Längle $F$, Wrba F, Ploder M et al (2006) Genetic detection of lymph node micrometastases: a selection criterion for liver transplantation in patients with liver metastases after colorectal cancer. Transplantation 81(1):64-70

18. Penn I (1991) Hepatic transplantation for primary and metastatic cancers of the liver. Surgery 110(4):726-734 (discussion 734-735)

19. Foss A, Adam R, Dueland S (2010) Liver transplantation for colorectal liver metastases: revisiting the concept. Transpl Int 23(7):679-685

20. Hoti E, Adam R (2008) Liver transplantation for primary and metastatic liver cancers. Transpl In 21(12):1107-1117

21. Hagness M, Foss A, Line PD, Scholz T, Jørgensen PF, Fosby B et al (2013) Liver transplantation for 
nonresectable liver metastases from colorectal cancer. Ann Surg 257(5):800-806. https://doi.org/ 10.1097/SLA.0b013e3182823957

22. D'Angelica M, Kornprat $\mathrm{P}$, Gonen $\mathrm{M}$, DeMatteo RP Fong $Y$, Blumgart LH et al (2011) Effect on outcome of recurrence patterns after hepatectomy for colorectal metastases. Ann Surg Oncol 18(4):1096-1103

23. Dueland S, Hagness M, Line PD, Guren TK, Tveit KM Foss A (2015) Is liver transplantation an option in colorectal cancer patients with nonresectable liver metastases and progression on all lines of standard chemotherapy? Ann Surg Oncol 22(7):2195-2200

24. Hagness M, Foss A, Egge TS, Dueland S (2014) Patterns of recurrence after liver transplantation for nonresectable liver metastases from colorectal cancer. Ann Surg Oncol 21(4):1323-1329

25. Dueland S, Guren TK, Hagness M, Glimelius B, Line PD, Pfeiffer P, Foss A, Tveit KM (2015) Chemotherapy or liver transplantation for nonresectable liver metastases from colorectal cancer? Ann Surg 261(5):956-960. https://doi.org/10.1097/ SLA.0000000000000786

26. Toso C, Pinto Marques $H$, Andres A, Castro Sousa F, Adam R, Kalil A et al (2017) Liver transplantation for colorectal liver metastasis: survival without recurrence can be achieved. Liver Transpl 23(8):1073-1076

27. Knight SR, Morris PJ, Schneeberger S, Pengel LH (2016) Trial design and endpoints in clinical transplant research. Transpl Int 29(8):870-879

28. Liu Q, Vekemans K, lania L, Komuta M, Parkkinen J, Heedfeld V et al (2014) Assessing warm ischemic injury of pig livers at hypothermic machine perfusion.J Surg Res 186(1):379-389

29. Karangwa SA, Burlage LC, Adelmeijer J, Karimian N Westerkamp AC, Matton AP et al (2017) Activation of fibrinolysis, but not coagulation, during end-Ischemic ex situ normothermic machine perfusion of human donor livers. Transplantation 101(2):e42-e48

30. op den Dries S, Karimian N, Sutton ME, Westerkamp AC, Nijsten MW, Gouw AS et al (2013) Ex vivo normothermic machine perfusion and viability testing of discarded human donor livers. Am J Transplant 13(5):1327-1335

31. Nasralla D, Coussios CC, Mergental H, Akhtar MZ, Butler AJ, Ceresa CDL et al (2018) A randomized trial of normothermic preservation in liver transplantation. Nature 557(7703):50-56

Hinweis des Verlags. Der Verlag bleibt in Hinblick auf geografische Zuordnungen und Gebietsbezeichnungen in veröffentlichten Karten und Institutsadressen neutral.

\section{Familiäres Darmkrebsrisiko: In den Genen steckt nur ein Teil der Wahrheit}

Wenn es um das familiär erhöhte Darmkrebsrisiko geht, wurde die Rolle der Gene überschätzt. Zu diesem Schluss kommen Wissenschaftler des Deutschen Krebsforschungszentrums (DKFZ) in einer aktuellen epidemiologischen Studie. Vermutlich wiegen andere Risikofaktoren wie etwa familiäre Ernährungsgewohnheiten schwerer als bislang angenommen. Das hat Konsequenzen für künftige Berechnungen des individuellen Risikos sowie auch für Empfehlungen, die daraus resultieren.

Darmkrebs ist die dritthäufigste Krebserkrankung weltweit. Wissenschaftler gehen derzeit davon aus, dass die Erkrankung zu 35 Prozent erblich bedingt ist. Zu den wichtigsten Risikofaktoren zählt eine familiäre Vorbelastung. Zudem haben Forschende in den letzten Jahren rund 100 winzige Genvariationen identifiziert, die in der Bevölkerung weit verbreitet sind (Einzelnukleotid-Polymorphismen, Single nucleodid polymorphisms, SNPs) und die das Risiko für Darmkrebs beeinflussen. Aktuelle Ergebnisse der DKFZ-Wissenschaftler um Hermann Brenner und Korbinian Weigl legen nun aber nahe, dass diese SNPs einen deutlich geringeren Anteil des familiär erhöhten Darmkrebsrisikos erklären als bisher angenommen.

Die DKFZ-Forscher haben zunächst die gängigen statistischen Verfahren geprüft, mit deren Hilfe dieser Anteil geschätzt wird. Dabei sind ihnen eine Reihe von Schwächen bei der Kalkulation aufgefallen, die in der Summe zu einer deutlichen Überschätzung führen. "Insbesondere gingen die bislang gängigen Verfahren davon aus, dass die familiäre Häufung letztendlich zu einhundert Prozent genetisch bedingt ist", sagt Korbinian Weigl, Erstautor der Studie. „Davon ausgehend berechneten sie dann, welcher Anteil hiervon durch die bislang bekannten Genvarianten erklärt werden kann."

Das Team um Brenner und Weigl wertete die Daten von 7.927 Probanden einer FallKontroll-Studie aus - darunter 4.447 Patienten mit Darmkrebs und 3.480 gesunde Personen, die als Kontrollgruppe dienten. "Wir haben eine neue Berechnungsmethode entwickelt, die nicht von vornherein von der Grundannahme ausgeht, dass das familiäre Risiko komplett durch die Genetik bedingt ist", erklärt Studienleiter Hermann Brenner. Mit dieser Methode werteten die Forscher dann die Daten ihrer Probanden aus. Tatsächlich war der Einfluss der SNPs nach dieser Methode wesentlich geringer als angenommen. Während bisherige Berechnungen den bislang bekannten Genvarianten einen Anteil von 9,6 bis 23,1 Prozent am familiär erhöhten Darmkrebsrisiko zusprachen, resultieren die neuen Berechnungen der DKFZForscher in einem geschätzten Einfluss der SNPs, der zwischen 5,4 und 14,3 Prozent liegt. „Die Ergebnisse passen sehr gut zu den Ergebnissen einer anderen DKFZ-Studie, die letztes Jahr publiziert wurde", erklärt Brenner. Dabei hatte sich gezeigt, dass bei Halbgeschwistern von Darmkrebspatienten das Risiko selbst zu erkranken ebenso erhöht ist wie bei „echten“ Geschwistern. „Das legt nahe, dass gemeinsame Risikofaktoren, etwa spezielle Ernährungsgewohnheiten, Rauchen oder ein Bewegungsmangel eine deutlich größere Rolle spielen als bisher angenommen."

Für die künftige Einschätzung des individuellen Darmkrebsrisikos bedeutet das aber auf keinen Fall, dass die Genvarianten, die mit einem erhöhten Risiko assoziiert werden, bedeutungslos geworden sind. „Vielmehr zeigt unsere Studie, wie wichtig es ist, sowohl genetische als auch andere Risikofaktoren in der Familie gleichermaßen zu betrachten, um zu einer realistischen Einschätzung zu gelangen", so Weigl.

Literatur Weigl K, Chang-Claude J, Hsu L, Hoffmeister M, Brenner H. Establishing a valid approach for estimating familial risk of cancer explained by common genetic variants. International Journal of Cancer 2019, DOI: $10.1002 /$ ijc.32664

Quelle: PM Deutsches Krebsforschungszentrum, 26.09.2019 